\title{
Cytoplasmic expression of C-MYC protein is associated with risk stratification of mantle cell lymphoma
}

\author{
Yi Gong ${ }^{1,2}$, Xi Zhang ${ }^{1}$, Rui Chen ${ }^{3}$, Yan Wei ${ }^{4}$, Zhongmin Zou ${ }^{\text {Corresp.. }}{ }^{5}$, Xinghua Chen ${ }^{\text {Corresp. } 1}$ \\ 1 Department of Hematology, Xinqiao Hospital, The Third Military Medical University, Chongqing, China \\ 2 Department of Hematology-oncology, Chongqing Cancer Institute/Hospital, Chongqing, China \\ 3 Department of Pathology, Chongqing Cancer Institute/Hospital, Chongqing, China \\ 4 Department of Pathology, Xinqiao Hospital, The Third Military Medical University, Chongqing, China \\ 5 Institute of Toxicology, School of Preventive Medicine, The Third Military Medical University, Chongqing, China \\ Corresponding Authors: Zhongmin Zou, Xinghua Chen \\ Email address: zouzhmin@yahoo.com, chenxh96@163.com
}

Aim: To investigate the association of C-MYC protein expression and risk stratification in mantle cell lymphoma (MCL), and to evaluate the utility of C-MYC protein as a prognostic biomarker in clinical practice.

Methods: We conducted immunohistochemical staining of C-MYC, Programmed cell death ligand 1 (PDL1), CD8, Ki-67, p53 and SRY (sex determining region Y) -11 (SOX11) to investigate their expression in 64 patients with $\mathrm{MCL}$. The staining results and other clinical data were evaluated for their roles in risk stratification of MCL cases using ANOVA, Chi-square, and Spearman's Rank correlation coefficient analysis.

Results: Immunohistochemical staining in our study indicated that SOX11, Ki-67 and p53 presented nuclear positivity of tumor cells, CD8 showed membrane positivity in infiltrating T lymphocytes while PDL1 showed membrane and cytoplasmic positivity mainly in macrophage cells and little in tumor cells. We observed positive staining of C-MYC either in the nucleus or cytoplasm or in both subcellular locations. There were significant differences in cytoplasmic C-MYC expression, Ki-67 proliferative index of tumor cells, and CD8 positive tumor infiltrating lymphocytes (CD8+TIL) among three risk groups $(P=0.000$, $P=0.037$ and $P=0.020$, respectively). However, no significant differences existed in the expression of nuclear C-MYC, SOX11, p53, and PD-L1 in MCL patients with low-, intermediate-, and high risks. In addition, patient age and serum LDH level were also significantly different among 3 groups of patients $(P=0.006$ and $P=0.000$, respectively). Spearman's rank correlation coefficient analysis indicated that cytoplasmic C-MYC expression, Ki-67 index, age, WBC, as well as LDH level had significantly positive correlations with risk stratification $(P=0.000,0.015,0.000,0.029$ and 0.000 , respectively), while CD8+TIL in tumor microenvironment negatively correlated with risk stratification of patients $(P=0.006)$. Patients with increased positive cytoplasmic expression of C-MYC protein and decreased CD8+TIL appeared to be associated with a poor response to chemotherapy, but the correlation was not statistically significant.

Conclusion: Our study suggested that assessment of cytoplasmic C-MYC overexpression and cytotoxic T lymphocytes (CTLs) by immunohistochemical staining might be helpful for MCL risk stratification and outcome prediction. However, large cohort studies of MCL patients with complete follow up are needed to validate our speculation. 
1 Title: Cytoplasmic expression of C-MYC protein is associated with risk stratification of mantle cell lymphoma

$2 \underline{\text { Yi Gong }}{ }^{1,2}, \underline{\text { Xi Zhang }}^{1}, \underline{\text { Rui Chen }}^{3}, \underline{\text { Yan Wei }}^{4}, \underline{\text { Zhongmin Zou }}^{5}, \underline{\text { Xinghua Chen }}^{1}$

3 'Department of Hematology, Xinqiao Hospital, the Third Military Medical University, Chongqing,

4 China

5 2Department of Hematology-oncology, Chongqing Cancer Institute/Hospital, Chongqing, China

63 Department of Pathology, Chongqing Cancer Institute/Hospital, Chongqing, China

7 4Department of Pathology, Xinqiao Hospital, the Third Military Medical University, Chongqing, China

8 5Institute of Toxicology, School of Preventive Medicine, the Third Military Medical University,

9 Chongqing, China

10

11

12

Abstract

Aim: To investigate the association of C-MYC protein expression and risk stratification in mantle cell lymphoma (MCL), and to evaluate the utility of C-MYC protein as a prognostic biomarker in clinical practice.

Methods: We conducted immunohistochemical staining of C-MYC, Programmed cell death ligand 1 (PD-L1), CD8, Ki-67, p53 and SRY (sex determining region Y) -11 (SOX11) to investigate their expression in 64 patients with MCL. The staining results and other clinical data were evaluated for their roles in risk stratification of MCL cases using ANOVA, Chi-square, and Spearman's Rank correlation coefficient analysis.

Results: Immunohistochemical staining in our study indicated that SOX11, Ki-67 and p53 presented nuclear positivity of tumor cells, CD8 showed membrane positivity in infiltrating T lymphocytes while PD-L1 showed membrane and cytoplasmic positivity mainly in macrophage cells and little in tumor cells. We observed positive staining of C-MYC either in the nucleus or cytoplasm or in both subcellular locations. There were significant differences in cytoplasmic C-MYC expression, Ki-67 proliferative index of tumor cells, and CD8 positive tumor infiltrating lymphocytes (CD8+TIL) among three risk groups $(P=0.000, P=0.037$ and $P=0.020$, respectively). However, no significant differences existed in the expression of nuclear C-MYC, SOX11, p53, and PD-L1 in MCL patients with low-, intermediate-, and high risks. In addition, patient age and serum LDH level were also significantly different among 3 groups of patients ( $P=0.006$ and $P=0.000$, respectively). Spearman's rank correlation coefficient analysis indicated that cytoplasmic C-MYC expression, Ki-67 index, age, WBC, as well as LDH level had significantly positive correlations with risk stratification $(P=0.000,0.015,0.000,0.029$ and 0.000, respectively), while CD8+TIL in tumor microenvironment negatively correlated with risk stratification of patients $(P=0.006)$. Patients with increased positive cytoplasmic expression of C-MYC protein and decreased CD8+TIL appeared to be associated with a poor response to chemotherapy, but the correlation was not statistically significant.

Conclusion: Our study suggested that assessment of cytoplasmic C-MYC overexpression and cytotoxic T lymphocytes (CTLS) by immunohistochemical staining might be helpful for MCL risk stratification and outcome prediction. However, large cohort studies of MCL patients with complete follow up are needed to validate our speculation.

\section{Introduction}

Mantle cell lymphoma (MCL) is a less frequent subtype of B cell non-Hodgkin lymphoma characterized by $\mathrm{t}(11 ; 14)$ chromosome translocation and aggressive clinical behavior(Smedby \& Hjalgrim 2011). Despite new 
advances of therapeutic methods in recent years, MCL remains an incurable disease with poor prognosis and most patients eventually succumb to relapse after initial therapy(CheahSeymour \& Wang 2016). CHOP (cyclophosphamide, doxorubicin, vincristine, and prednisone)-like chemotherapy with or without rituximab or autologous stem cell transplantation (ASCT) is the most popular regimen applied for the treatment of MCL, with complete remission rates of $20 \%-50 \%$ and a median overall survival of about 3 years(Dreyling 2014; Vose 2015). Dose-intensified chemotherapy such as Hyper-CVAD (hyperfractionated cyclophosphamide, vincristine, doxorubicin, and dexamethasone alternating with high-dose methotrexate and cytarabine) with rituximab has achieved higher complete remission rates and 3-year failure-free survival in several clinical studies, however, it has also induced significant toxicity, which made it ineligible for most elderly and frail patients(KhouriRomagueraKantarjianPalmerPughKorblingHagemeisterSamuelsRodriguez \& Giralt 2016; RomagueraFayadRodriguezBroglioHagemeisterProMclaughlinYounesSamaniego \& Goy 2005). In recent years, with the advance in molecular pathogenesis of $\mathrm{MCL}$, novel small molecular drugs including Bortezomib, Lenalidomide, ibrutinib, and idelalisib have been introduced into treatment of relapsed/refractory MCL and shown promising clinical outcome for part of these patients(InamdarGoyAyoubAttiaOtonTaruvaiCostalesLinPecora \& Suh 2016b). Due to genetic, pathological and clinical heterogeneity of MCL (InamdarGoyAyoubAttiaOtonTaruvaiCostalesLinPecora \& Suh 2016a), there is a critical need for reproducible biomarkers to guide the decision of individualized risk-adapted treatment for MCL patients.

C-MYC is one of the most frequently deregulated oncogenes in human cancer, and C-MYC encoded protein functions as an important transcription factor involved in the regulation of cell growth and cell cycle progression (DangO'DonnellZellerNguyenOsthus \& Li 2006; Sears 2004). In malignant cells, genetic alteration on C-MYC gene leads to consistent overexpression of C-MYC protein and promotes tumor progression (Meyer \& Penn 2008; Ott 2014). High expression of C-MYC was reported to be associated with poor outcome of a large quantity of malignant diseases including aggressive lymphomas (SewastianikProchorec-SobieszekChapuy \& Juszczynski 2014). C-MYC abnormality has also been found in MCL patients with worse prognosis (ChoeYunNaHuhShinKimPaikKimNamJeonPark $\quad \& \quad$ Kim 2016; HuMedeirosChenChenLiKonoplevLuPhamYoungWang \& Hu 2017). Recently, the immune-modulatory function of C-MYC was identified in tumorigenesis (CaseyBaylot \& Felsher 2017; CaseyTongLiDoWalzFitzgeraldGouwBaylotGutgemannEilers \& Felsher 2016), which has drawn much attention on the role of oncogenes in tumor microenvironment.

Mantle cell lymphoma international prognostic index (MIPI) has been widely used for risk stratification of advanced MCL patients. It combines clinical and laboratory parameters to divide patients into low-, intermediate-, and high-risk groups (HosterDreylingKlapperGisselbrechtvan HoofKluinNelemansPfreundschuhReiserMetznerEinselePeterJungWormannLudwigDuhrsenEimermacherWandtHasford Hiddemann \& Unterhalt 2008; HosterKlapperHermineKluin-NelemansWalewskivan HoofTrnenyGeislerDi RaimondoSzymczykStilgenbauerThieblemontHallekForstpointnerPottRibragDoorduijnHiddemannDreyling \& Unterhalt 2014). However, the correlation between C-MYC expression and MIPI, and the clinical value of CMYC expression in treatment decision for MCL patients are not clear. In this study, we investigated the potential of C-MYC protein level assessed by immunohistochemistry for MCL risk stratification and evaluated its role in individualized therapies for patients. 


\section{Materials and methods Case selection}

Cases of MCL diagnosed between 2013 and 2016 in Chongqing Cancer Institute/Hospital were reviewed for complete information including pathological tissues and in-patient history. Sixty-four cases were identified and included in this study. All cases were reviewed by two experienced pathologists according to the criteria of the World Health Organization Classification. Data on clinical and laboratory findings such as staging, white blood cell (WBC) count, renal and liver function tests including serum albumin, globulin and 32 -microglobulin $(\beta 2 \mathrm{M})$, bone marrow biopsy, and imaging examinations (ultrasonic examination and radiologic examination of brain, chest, abdomen and pelvis) at the time of diagnosis were reviewed. Patients were staged according to Ann Arbor classification, and risk stratification was performed based on MIPI score system. Evaluation of therapy response was based on the Lugano classification for response assessment of Hodgkin and non-Hodgkin Iymphoma(ChesonFisherBarringtonCavalliSchwartzZucca \& Lister 2014). All the cases were divided into low-, intermediate-, and high-risk groups according to MIPI score, as well as complete remission (CR), partial remission (PR) and progressive disease (PD) groups according to the therapy response. The study was approved by the ethics committees of Chongqing Cancer Institute/Hospital (No. 2017-016).

\section{Immunohistochemistry analysis}

Immunohistochemical staining was performed using formalin-fixed paraffin-embedded tissue sections from pre-therapeutic samples according to the manufacturer's instructions. Rabbit anti-human C-MYC monoclonal antibody (clone Y69, diluted in 1:150), mouse anti-human Ki-67 monoclonal antibody (clone MIB1, diluted in 1:200), and rabbit anti-human PD-L1 monoclonal antibody (clone SP142, diluted in 1:150) were purchased from ORIGENE (USA). Rabbit anti-human CD8 monoclonal antibody (clone SP16, diluted in 1:200), mouse anti-human SOX11 monoclonal antibody (clone MRQ-58, diluted in 1:150), and rabbit anti-human p53 monoclonal antibody (clone SP5, diluted in 1:200) were purchased from Abcam (USA). GTVision III detection system was purchased from DAKO (USA). Positivity of membranous and cytoplasmic staining pattern was scored by the staining density, ranging from 0 to 3 ( $0=$ no staining/-, 1=weak staining/+, 2=moderate staining/++, $3=$ strong staining/+++). Positivity of nuclear staining pattern was quantified as the percentage of positive $\mathrm{MCL}$ cells by manual inspection of stained sections. Tumor-infiltrating CD8 positive $T$ cells were quantified as total counts of CD8 positive lymphocytes per high power field (HPF; $0.2 \mathrm{~mm}^{2}$ ) by manual inspection of stained sections with at least 10 fields of strong staining density. All the immuno-staining sections were independently determined by 2 experienced pathologists in a blinded fashion.

\section{Statistical analysis}

All data were analyzed with SPSS 18.0 (IBM Corporation, NY, USA). Categorical variables were compared using chi-square test. The difference between continuous variables was assessed using ANOVA. The role of above clinicopathological parameters for $\mathrm{MCL}$ risk stratification and therapy individualization were evaluated based on MIPI score and clinical response respectively. $P$ value of less than 0.05 was considered statistically significant. Spearman's rank correlation coefficient analysis was performed to assess the association between clinical factors and immunohistochemical staining results of C-MYC, Ki-67, PD-L1, CD8, SOX11 and p53.

\section{Results}




\section{Patient characteristics}

There were 51 male (79.7\%) and 13 female (20.3\%) patients. The median age was 60 years old (range 2187 years) at the time of diagnosis. The majority of the cases (71.9\%) presented as nodal diseases, and 18 cases occurred in primary extra-nodal sites including colon, rectum, ileocecum, oropharynx, and spleen. Sixty-one (95.3\%) patients were in Ann Arbor advanced stage (III-IV) and 35 (54.7\%) patients presented with B symptoms. Only 4 (6.4\%) patients had an ECOG performance status (PS) of 2-4. Thirty-one of the 64 patients had treatment information of at least 2 cycles of chemotherapy for response evaluation. As depicted in supplementary data Table 1, 13 patients received CHOP, 11 patients received rituximab combined with $\mathrm{CHOP}$, 3 patients received CHOPE, 3 patients received Hyper-CVAD and 1 patients received GDP treatment. After 2 treatment cycles, 5 patients achieved CR, 17 patients achieved PR and 9 patients demonstrated PD.

\section{Immunohistochemical study}

As shown in Figure 1, SOX11, Ki-67 and p53 presented nuclear positivity of tumor cells, and CD8 showed membrane positivity of T lymphocytes infiltrating the microenvironment. PD-L1 showed membrane and cytoplasm positive pattern mainly in macrophage cells and little in tumor cells. However, C-MYC staining was observed either in the nucleus or cytoplasm or in both subcellular locations.

As shown in Table 1, there were significant differences in cytoplasmic C-MYC expression, Ki-67 proliferative index of tumor cells, and CD8 positive tumor infiltrating lymphocytes (CD8+TIL) among three risk groups ( $P=0.000, P=0.037, P=0.020$ ). However, no significant difference existed in the expression of nuclear $\mathrm{C}-$ MYC, SOX11, p53, and PD-L1 among MCL patients with low, intermediate, and high risks, respectively. In addition, other clinical parameters including age and LDH level showed significant difference among 3 groups of patients as defined by MIPI score $(P=0.006, P=0.000)$.

Spearman's rank correlation coefficient analysis was performed to evaluate the relationship between clinicopathologcial parameters and prognosis or therapy response. As shown in Table 1, patients' age, WBC, LDH level, as well as cytoplasmic C-MYC expression and Ki-67 index demonstrated a significantly positive correlation with risk stratification $(P=0.000,0.029,0.000$, and 0.000 , respectively), while CD8+TIL in tumor microenvironment negatively correlated with risk stratification of patients $(P=0.006)$. Increased positive cytoplasmic expression of C-MYC protein and decreased CD8+TIL were associated with a poor response to chemotherapy, but the correlation did not reach statistical significance (Table 2 and Table 3 ).

\section{Discussion}

Assessment of C-MYC oncogene condition is critical for differentiating diagnosis and predicting prognosis in Burkitt lymphoma and diffuse large B cell lymphoma harboring a C-MYC translocation(DallaFaveraBregniEriksonPattersonGallo \& Croce 1982; OttRosenwald \& Campo 2013; SavageJohnsonBenNeriahConnorsSehnFarinhaHorsman \& Gascoyne 2009). As a potent nuclear transcription factor, C-MYC protein overexpression has been typically found in the nucleus of lymphoma cells(ChoeYunNaHuhShinKimPaikKimNam \& Jeon 2016; OberleyRajguruZhangKimShawGrindleKahIKanughLaffin \& Yang 2013). However, in this study, we observed three patterns of C-MYC expression including nuclear, cytoplasmic, and both nuclear and cytoplasmic localization in mantle cell lymphoma (Figure 1), which was different from the results reported by Matthew J. 
et al using commercially available C-MYC monoclonal antibody (clone number: Y69). The immunohistochemistry staining procedures have been independently validated by two experienced pathologists to exclude false positive or non-specific results. We searched a few studies that reported cytoplasmic expression of C-MYC in leukemia cell line, endometrial carcinoma, and high grade B cell lymphomas (CraigBuchanCivin \& Kastan 1993; GeislerGeislerManahanMillerWiemannZhou \& Crabtree 2004; RuzinovaCaron \& Rodig 2010), but all the authors defined the cytoplasmic C-MYC status as negative or positive, which was different with our four-categories evaluation methods $(0,+,++,+++)$. The mechanism and biologic importance for the cytoplasmic overexpression of C-MYC was unclear. Since C-MYC needs to dimerize with Max to bind the E-box to activate its downstream genes in transformed cells(Dang et al. 2006), accumulation of C-MYC protein in the cytoplasm might suggest an unknown deregulated pathway synergized with other pathways in promoting tumor growth. Therefore, the cytoplasmic pattern of C-MYC expression might indicate a worse prognosis compared with the typical nuclear pattern. In this study, we found a positive correlation only between cytoplasmic C-MYC level and risk stratification of MCL, but not between nuclear C-MYC level and risk stratification of $\mathrm{MCL}$, suggesting the potential clinical application of C-MYC immunohistochemical staining in determining prognosis and treatment of MCL patients.

PD-L1 is an important immune checkpoint molecule in tumor microenvironment, which is associated with immune evasion in a variety of malignancies (Dong \& Chen 2003; JanakiramPareekChengNarasimhulu \& Zang 2016; WlasiukPutowski \& Giannopoulos 2016). Prevalence of Th1 type lymphocytes such as CD8+ T lymphocytes usually predicts good prognosis of cancer patients (DonnemHaldPaulsenRichardsenAlSaadKilvaerBrustugunHellandLund-IversenPoehIOlsenDitzelHansenAlShiblikiselevSandangerAndersenPezzellaBremnes \& Busund 2015; Hanahan \& Weinberg 2011). Expression of PD-L1 and infiltration of cytotoxic T lymphocytes (CTLS) in tumor microenvironment are reported to be the prerequisite for effective response of PD-L1 pathway blockade therapy in many cancer patients (OckKeamKimLeeKimKimJeonKimChung \& Heo 2016; TengNgiowRibas \& Smyth 2015). High level of PD-L1 expression has been observed in some types of lymphomas including a subset of aggressive B cell lymphomas and EBV-associated malignancies such as classical Hodgkin lymphoma (CHL)(BerghoffRickenWidhalmRajkyHainfellnerBirnerRaderer \& $\quad$ Preusser $\quad$ 2014; ChenChapuyOuyangSunRoemerXuYuFletcherFreemanShipp \& $\quad$ Rodig 2013; KwonKimKimGoNamPaikKimKimHeoKim \& Jeon 2016). In our study, positive PD-L1 staining was found in $68.75 \%$ (33/48) MCL patients but only 1.04\% (5/48) cases were recorded as moderate to strong positivity. In addition, PD-L1 positive cells were mainly macrophages in the microenvironment. There was no significant difference in PD-L1 expression among various risk groups, but the density of CD8+ T lymphocytes showed a negative correlation with risk stratification, suggesting that CD8+ T lymphocytes might be a useful prognostic biomarker for risk stratification of $\mathrm{MCL}$ patients, consistent with other previous studies (NygrenWasikBaumgartner-WennerholmJeppsson-

AhlbergKlimkowskaAnderssonBuhrkuhlChristenssonKimbyWahlin \& Sander 2014). Extensive future study of PD1/PD-L1 pathway in a large cohort of MCL patients is also warranted.

$\mathrm{Ki}-67$ as an independent, significant prognostic factor for MCL has been proved in many clinical studies and integrated into MIPI score system as a combined biological index (MIPI $\mathrm{b}_{b}$ )(Hoster et al. 2008). The significance of Ki-67 was also confirmed in our study. We similarly assessed the expression of another two important biomarkers, SOX11(EkDictorJerkemanJirstrom \& Borrebaeck 2008; Xu \& Li 2010) and p53 
206

(NordstromSernboEdenGronbaekKolstadRatyKarjalainenGeislerRalfkiaerSundstromLaurellDelabieEhingerJerk eman \& Ek 2014; TessoulinEveillardLokChironMoreauAmiotMoreau-AubryLe Gouill \& Pellat-Deceunynck 2017), in MCL cases but found no significant difference among low, intermediate, and high risk group of $M C L$ patients.

We also noted that patients with increased positive cytoplasmic expression of C-MYC protein and decreased CD8+TIL were associated with poor response to chemotherapy, but the correlation did not reach statistical significance, probably due to the loss of follow-up date in 33 of 64 patients in our cohort.

Limitations of the study included small cohort of cases and insufficient survival data because of the difficulties in follow-up for most lymphoma patients of this area. In addition, the systematic bias including tissue fixation and observation might influence the accuracy of assessment for protein biomarkers in immunohistochemistry studies (TorlakovicRiddellBanerjeeElZimaityPilavdzicDaweMaglioccoBarnesBerendtCookGilksWilliamsPerezOrdonezWehrliSwansonOtisNielsenVyberg \& Butany 2010). Nevertheless, this is the first report on the association of aberrant expression of C-MYC protein, $\mathrm{CD} 8+\mathrm{TIL}$, and Ki- 67 with risk stratification of $\mathrm{MCL}$ patients.

In conclusion, our work suggested that assessment of cytoplasmic C-MYC overexpression, CD8 positive CTLs, and Ki- 67 by immunohistochemical staining might be helpful for risk stratification and prognosis of $\mathrm{MCL}$ patients. Large cohort studies of MCL patients with complete follow up are needed to further examine the potential of these biomarkers being integrated into routine pathological work.

\section{References}

Berghoff AS, Ricken G, Widhalm G, Rajky O, Hainfellner JA, Birner P, Raderer M, and Preusser M. 2014. PD1 (CD279) and PD-L1 (CD274, B7H1) expression in primary central nervous system lymphomas (PCNSL). Clin Neuropathol 33:42-49.

Casey SC, Baylot V, and Felsher DW. 2017. MYC: Master Regulator of Immune Privilege. Trends Immunol. 10.1016/j.it.2017.01.002

Casey SC, Tong L, Li Y, Do R, Walz S, Fitzgerald KN, Gouw AM, Baylot V, Gutgemann I, Eilers M, and Felsher DW. 2016. MYC regulates the antitumor immune response through CD47 and PD-L1. Science 352:227-231. 10.1126/science.aac9935

Cheah CY, Seymour JF, and Wang ML. 2016. Mantle Cell Lymphoma. J Clin Oncol 34:1256-1269. 10.1200/jco.2015.63.5904

Chen BJ, Chapuy B, Ouyang J, Sun HH, Roemer MG, Xu ML, Yu H, Fletcher CD, Freeman GJ, Shipp MA, and Rodig SJ. 2013. PD-L1 expression is characteristic of a subset of aggressive B-cell lymphomas and virus-associated malignancies. Clin Cancer Res 19:3462-3473. 10.1158/1078-0432.ccr-13-0855

Cheson BD, Fisher RI, Barrington SF, Cavalli F, Schwartz LH, Zucca E, and Lister TA. 2014. Recommendations for Initial Evaluation, Staging, and Response Assessment of Hodgkin and Non-Hodgkin Lymphoma: The Lugano Classification. Journal of Clinical Oncology 32:3059-3067.

Choe J, Yun JY, Na HY, Huh J, Shin S, Kim H, Paik JH, Kim YA, Nam SJ, and Jeon YK. 2016. MYC overexpression correlates with MYC amplification or translocation, and is associated with poor prognosis in mantle cell lymphoma. Histopathology 68:442-449. 
247

248

249

250

251

252

253

254

255

256

257

258

259

260

261

262

263

264

265

266

267

268

269

270

271

272

273

274

275

276

277

278

279

280

281

282

283

284

285

286

287

Choe JY, Yun JY, Na HY, Huh J, Shin SJ, Kim HJ, Paik JH, Kim YA, Nam SJ, Jeon YK, Park G, and Kim JE. 2016. MYC overexpression correlates with MYC amplification or translocation, and is associated with poor prognosis in mantle cell lymphoma. Histopathology 68:442-449. 10.1111/his.12760

Craig RW, Buchan HL, Civin Cl, and Kastan MB. 1993. Altered cytoplasmic/nuclear distribution of the c-myc protein in differentiating ML-1 human myeloid leukemia cells. Cell Growth Differ 4:349-357.

Dalla-Favera R, Bregni M, Erikson J, Patterson D, Gallo RC, and Croce CM. 1982. Human c-myc onc gene is located on the region of chromosome 8 that is translocated in Burkitt lymphoma cells. Proc Natl Acad Sci U S A 79:78247827.

Dang CV, O'Donnell KA, Zeller KI, Nguyen T, Osthus RC, and Li F. 2006. The c-Myc target gene network. Semin Cancer Biol 16:253-264. 10.1016/j.semcancer.2006.07.014

Dong H, and Chen L. 2003. B7-H1 pathway and its role in the evasion of tumor immunity. J Mol Med (Berl) 81:281287. 10.1007/s00109-003-0430-2

Donnem T, Hald SM, Paulsen EE, Richardsen E, Al-Saad S, Kilvaer TK, Brustugun OT, Helland A, Lund-Iversen M, Poehl M, Olsen KE, Ditzel HJ, Hansen O, Al-Shibli K, Kiselev Y, Sandanger TM, Andersen S, Pezzella F, Bremnes RM, and Busund LT. 2015. Stromal CD8+ T-cell Density-A Promising Supplement to TNM Staging in Non-Small Cell Lung Cancer. Clin Cancer Res 21:2635-2643. 10.1158/1078-0432.ccr-14-1905

Dreyling M. 2014. Mantle cell lymphoma: biology, clinical presentation, and therapeutic approaches. Am Soc Clin Oncol Educ Book:191-198. 10.14694/EdBook_AM.2014.34.191

Ek S, Dictor M, Jerkeman M, Jirstrom K, and Borrebaeck CA. 2008. Nuclear expression of the non B-cell lineage Sox11 transcription factor identifies mantle cell lymphoma. Blood 111:800-805. 10.1182/blood-2007-06-093401

Geisler JP, Geisler HE, Manahan KJ, Miller GA, Wiemann MC, Zhou Z, and Crabtree W. 2004. Nuclear and cytoplasmic c-myc staining in endometrial carcinoma and their relationship to survival. Int J Gynecol Cancer 14:133-137.

Hanahan D, and Weinberg RA. 2011. Hallmarks of cancer: the next generation. Cell 144:646-674. 10.1016/j.cell.2011.02.013

Hoster E, Dreyling M, Klapper W, Gisselbrecht C, van Hoof A, Kluin-Nelemans HC, Pfreundschuh M, Reiser M, Metzner B, Einsele H, Peter N, Jung W, Wormann B, Ludwig WD, Duhrsen U, Eimermacher H, Wandt H, Hasford J, Hiddemann W, and Unterhalt M. 2008. A new prognostic index (MIPI) for patients with advanced-stage mantle cell lymphoma. Blood 111:558-565. 10.1182/blood-2007-06-095331

Hoster E, Klapper W, Hermine O, Kluin-Nelemans HC, Walewski J, van Hoof A, Trneny M, Geisler CH, Di Raimondo F, Szymczyk M, Stilgenbauer S, Thieblemont C, Hallek M, Forstpointner R, Pott C, Ribrag V, Doorduijn J, Hiddemann W, Dreyling $\mathrm{MH}$, and Unterhalt M. 2014. Confirmation of the mantle-cell lymphoma International Prognostic Index in randomized trials of the European Mantle-Cell Lymphoma Network. J Clin Oncol 32:1338-1346. 10.1200/jco.2013.52.2466

Hu Z, Medeiros L, Chen Z, Chen W, Li S, Konoplev SN, Lu X, Pham LV, Young KH, Wang W, and Hu S. 2017. Mantle Cell Lymphoma With MYC Rearrangement: A Report of 17 Patients. Am J Surg Pathol 41:216-224. 10.1097/pas.0000000000000758

Inamdar AA, Goy A, Ayoub NM, Attia C, Oton L, Taruvai V, Costales M, Lin YT, Pecora A, and Suh KS. 2016a. Mantle cell lymphoma in the era of precision medicine-diagnosis, biomarkers and therapeutic agents. Oncotarget 7:48692-48731. 10.18632/oncotarget.8961

Inamdar AA, Goy A, Ayoub NM, Attia C, Oton LF, Taruvai V, Costales M, Lin Y, Pecora AL, and Suh KS. 2016b. Mantle cell lymphoma in the era of precision medicine-diagnosis, biomarkers and therapeutic agents. Oncotarget 
7:48692-48731.

Janakiram M, Pareek V, Cheng H, Narasimhulu DM, and Zang X. 2016. Immune checkpoint blockade in human cancer therapy: lung cancer and hematologic malignancies. Immunotherapy 8:809-819. 10.2217/imt-2016-0001

Khouri IF, Romaguera J, Kantarjian H, Palmer J, Pugh W, Korbling M, Hagemeister FB, Samuels BI, Rodriguez A, and Giralt S. 2016. Hyper-CVAD and high-dose methotrexate/cytarabine followed by stem-cell transplantation: an active regimen for aggressive mantle-cell lymphoma. Journal of Clinical Oncology 16:3803-3809.

Kwon D, Kim S, Kim PJ, Go H, Nam SJ, Paik JH, Kim YA, Kim TM, Heo DS, Kim CW, and Jeon YK. 2016. Clinicopathological analysis of programmed cell death 1 and programmed cell death ligand 1 expression in the tumour microenvironments of diffuse large B cell lymphomas. Histopathology 68:1079-1089. 10.1111/his.12882

Meyer N, and Penn LZ. 2008. Reflecting on 25 years with MYC. Nat Rev Cancer 8:976-990. 10.1038/nrc2231

Nordstrom L, Sernbo S, Eden P, Gronbaek K, Kolstad A, Raty R, Karjalainen ML, Geisler C, Ralfkiaer E, Sundstrom C, Laurell A, Delabie J, Ehinger M, Jerkeman M, and Ek S. 2014. SOX11 and TP53 add prognostic information to MIPI in a homogenously treated cohort of mantle cell lymphoma--a Nordic Lymphoma Group study. $\mathrm{Br} J$ Haematol 166:98-108. 10.1111/bjh.12854

Nygren L, Wasik AM, Baumgartner-Wennerholm S, Jeppsson-Ahlberg A, Klimkowska M, Andersson P, Buhrkuhl D, Christensson B, Kimby E, Wahlin BE, and Sander B. 2014. T-cell levels are prognostic in mantle cell lymphoma. Clin Cancer Res 20:6096-6104. 10.1158/1078-0432.ccr-14-0889

Oberley MJ, Rajguru S, Zhang C, Kim K, Shaw GR, Grindle KM, Kahl BS, Kanugh C, Laffin JJ, and Yang DT. 2013. Immunohistochemical evaluation of MYC expression in mantle cell lymphoma. Histopathology 63:499-508.

Ock CY, Keam B, Kim S, Lee JS, Kim M, Kim TM, Jeon YK, Kim DW, Chung DH, and Heo DS. 2016. Pan-Cancer Immunogenomic Perspective on the Tumor Microenvironment Based on PD-L1 and CD8 T-Cell Infiltration. Clin Cancer Res 22:2261-2270. 10.1158/1078-0432.ccr-15-2834

Ott G. 2014. Impact of MYC on malignant behavior. Hematology Am Soc Hematol Educ Program 2014:100-106. 10.1182/asheducation-2014.1.100

Ott G, Rosenwald A, and Campo E. 2013. Understanding MYC-driven aggressive B-cell lymphomas: pathogenesis and classification. Blood 122:3884-3891. 10.1182/blood-2013-05-498329

Romaguera J, Fayad L, Rodriguez MA, Broglio K, Hagemeister FB, Pro B, Mclaughlin P, Younes A, Samaniego F, and Goy A. 2005. High Rate of Durable Remissions After Treatment of Newly Diagnosed Aggressive Mantle-Cell Lymphoma With Rituximab Plus Hyper-CVAD Alternating With Rituximab Plus High-Dose Methotrexate and Cytarabine. Journal of Clinical Oncology 23:7013-7023.

Ruzinova MB, Caron T, and Rodig SJ. 2010. Altered subcellular localization of c-Myc protein identifies aggressive Bcell lymphomas harboring a C-MYC translocation. Am J Surg Pathol 34:882-891. 10.1097/PAS.0b013e3181db83af

Savage KJ, Johnson NA, Ben-Neriah S, Connors JM, Sehn LH, Farinha P, Horsman DE, and Gascoyne RD. 2009. MYC gene rearrangements are associated with a poor prognosis in diffuse large B-cell lymphoma patients treated with R-CHOP chemotherapy. Blood 114:3533-3537. 10.1182/blood-2009-05-220095

Sears RC. 2004. The life cycle of C-myc: from synthesis to degradation. Cell Cycle 3:1133-1137.

Sewastianik T, Prochorec-Sobieszek M, Chapuy B, and Juszczynski P. 2014. MYC deregulation in lymphoid tumors: molecular mechanisms, clinical consequences and therapeutic implications. Biochim Biophys Acta 1846:457-467. 10.1016/j.bbcan.2014.08.006

Smedby KE, and Hjalgrim H. 2011. Epidemiology and etiology of mantle cell lymphoma and other non-Hodgkin 
329

330

331

332

333

334

335

336

337

338

339

340

341

342

343

344

345

lymphoma subtypes. Semin Cancer Biol 21:293-298. 10.1016/j.semcancer.2011.09.010

Teng MW, Ngiow SF, Ribas A, and Smyth MJ. 2015. Classifying Cancers Based on T-cell Infiltration and PD-L1. Cancer Res 75:2139-2145. 10.1158/0008-5472.can-15-0255

Tessoulin B, Eveillard M, Lok A, Chiron D, Moreau P, Amiot M, Moreau-Aubry A, Le Gouill S, and Pellat-Deceunynck C. 2017. p53 dysregulation in B-cell malignancies: More than a single gene in the pathway to hell. Blood Rev. 10.1016/j.blre.2017.03.001

Torlakovic EE, Riddell R, Banerjee D, El-Zimaity H, Pilavdzic D, Dawe P, Magliocco A, Barnes P, Berendt R, Cook D, Gilks B, Williams G, Perez-Ordonez B, Wehrli B, Swanson PE, Otis CN, Nielsen S, Vyberg M, and Butany J. 2010. Canadian Association of Pathologists-Association canadienne des pathologistes National Standards Committee/Immunohistochemistry: best practice recommendations for standardization of immunohistochemistry tests. Am J Clin Pathol 133:354-365. 10.1309/ajcpdyz1xmf4hjwk

Vose JM. 2015. Mantle cell lymphoma: 2015 update on diagnosis, risk-stratification, and clinical management. Am J Hematol 90:739-745. 10.1002/ajh.24094

Wlasiuk P, Putowski M, and Giannopoulos K. 2016. PD1/PD1L pathway, HLA-G and T regulatory cells as new markers of immunosuppression in cancers. Postepy Hig Med Dosw (Online) 70:1044-1058.

Xu W, and Li JY. 2010. SOX11 expression in mantle cell lymphoma. Leuk Lymphoma 51:1962-1967. $10.3109 / 10428194.2010 .514968$ 


\section{Figure 1}

Immunohistochemical staining of mantle cell lymphoma using anti-SOX11, Ki67, p53, CMYC,CD8, PD-L1

Shown are representative staining patterns of S0X11 (A), Ki-67 (B), p53 (C), C-MYC (D-F),CD8 (G-I), PD-L1 (J-L). Original magnification, $\times 200$. Inserts: typical cytoplasmic and nuclear staining of C-MYC (D), nuclear staining of C-MYC (E), and cytoplasmic staining of C-MYC (F), Original magnification, $\times 400$.
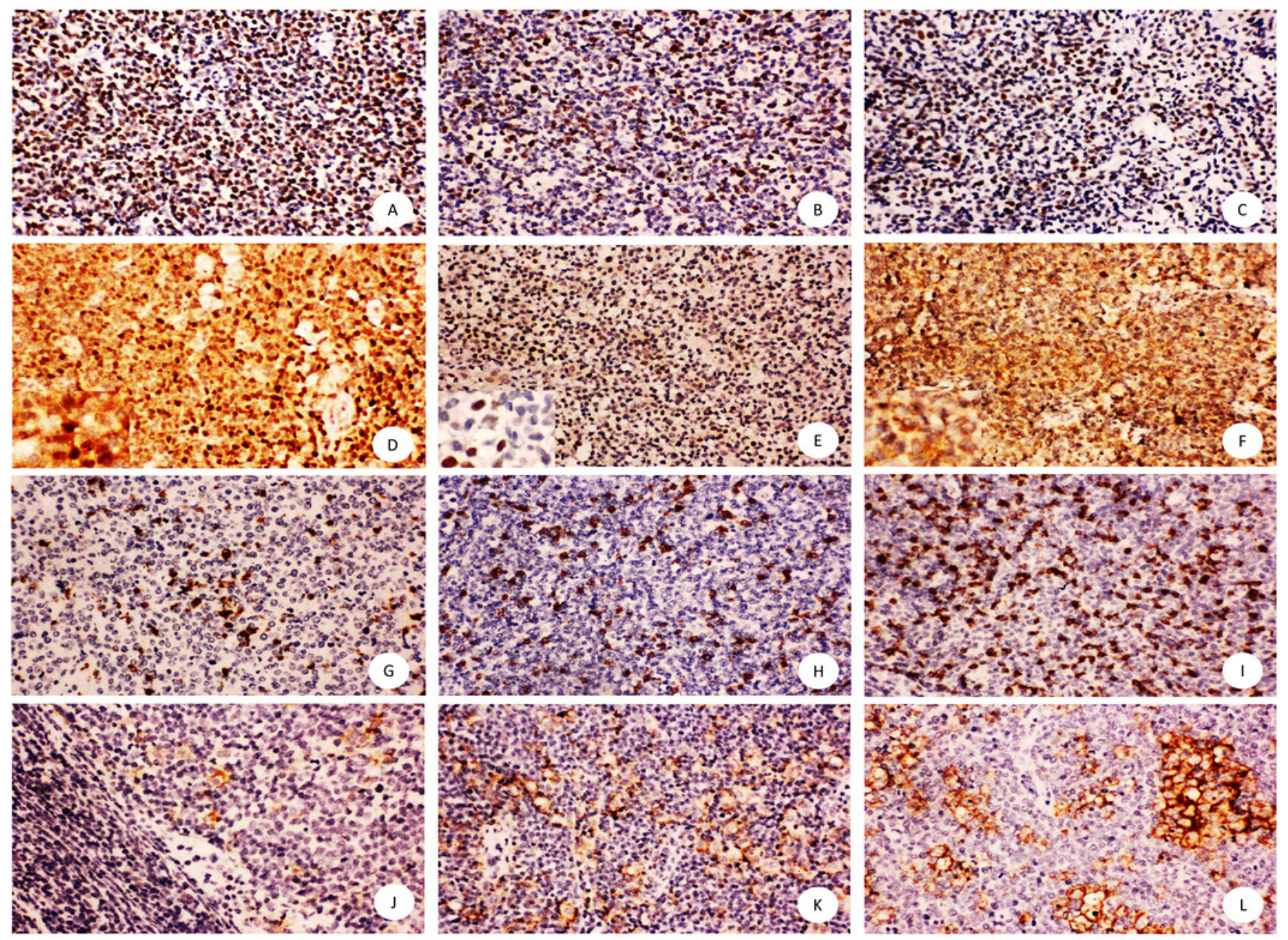


\section{Table $\mathbf{1}$ (on next page)}

Clinical and immunohistochemical staining characteristics of patients grouped by risk

Note: $* P<0.05, * * P<0.01$. 


\begin{tabular}{|c|c|c|c|c|c|}
\hline Variable & $\mathbf{n}$ & Low risk & $\begin{array}{c}\text { Intermediate } \\
\text { risk }\end{array}$ & High risk & $\begin{array}{c}P \\
\text { value }\end{array}$ \\
\hline $\operatorname{Sex}$ & 64 & & & & .117 \\
\hline Male & $51(79.7 \%)$ & $15(23.4 \%)$ & $27(42.2 \%)$ & $9(14.1 \%)$ & \\
\hline Female & $13(20.3 \%)$ & $5(7.8 \%)$ & $3(4.7 \%)$ & $5(7.8 \%)$ & \\
\hline Age (years) & 64 & $54.10 \pm 8.60$ & $61.77 \pm 7.77$ & $64.86 \pm 15.39$ & $.006^{* *}$ \\
\hline$W B C\left(10^{9} / L\right)$ & 64 & $6.50 \pm 2.17$ & $8.07 \pm 3.69$ & $8.93 \pm 3.19$ & .078 \\
\hline$L D H(U / L)$ & 64 & $190.03 \pm 74.84$ & $341.35 \pm 226.03$ & $521.15 \pm 266.44$ & $.000^{* *}$ \\
\hline ECOG performance & 64 & & & & 0.112 \\
\hline \multicolumn{6}{|l|}{ Status } \\
\hline $\mathbf{0}$ & $46(71.9 \%)$ & $16(25.0 \%)$ & $22(34.4 \%)$ & $8(12.5 \%)$ & \\
\hline 1 & $14(21.9 \%)$ & $4(6.3 \%)$ & $7(10.9 \%)$ & $3(4.7 \%)$ & \\
\hline 2 & $4(6.2 \%)$ & $0(0.0 \%)$ & $1(1.6 \%)$ & $3(4.7 \%)$ & \\
\hline Ann Arbor stage & 64 & & & & .302 \\
\hline II & $3(4.69 \%)$ & $2(3.1 \%)$ & $1(1.6 \%)$ & $0(0.0 \%)$ & \\
\hline III & $32(50.0 \%)$ & $11(17.2 \%)$ & $12(18.8 \%)$ & $9(14.1 \%)$ & \\
\hline IV & $29(45.31 \%)$ & $7(10.9 \%)$ & $17(26.6 \%)$ & $5(7.8 \%)$ & \\
\hline Location & 64 & & & & 0.589 \\
\hline Nodal & $46(71.9 \%)$ & $16(25.0 \%)$ & $20(31.3 \%)$ & $10(15.6 \%)$ & \\
\hline Extra-nodal & $18(28.1 \%)$ & $4(6.3 \%)$ & $10(15.6 \%)$ & $4(6.3 \%)$ & \\
\hline B symptoms & 64 & & & & .363 \\
\hline No & $29(45.3 \%)$ & $10(15.6 \%)$ & $15(23.4 \%)$ & $4(6.3 \%)$ & \\
\hline Yes & $35(54.7 \%)$ & $10(15.6 \%)$ & $15(23.4 \%)$ & $10(15.6 \%)$ & \\
\hline Response & 31 & & & & .883 \\
\hline $\mathrm{CR}$ & $5(7.8 \%)$ & $2(6.5 \%)$ & $3(9.7 \%)$ & $0(0.0 \%)$ & \\
\hline PR & $17(26.56 \%)$ & $6(19.4 \%)$ & $8(25.8 \%)$ & $3(9.7 \%)$ & \\
\hline PD & $9(14.1 \%)$ & $3(9.7 \%)$ & $5(16.1 \%)$ & $1(3.2 \%)$ & \\
\hline Cytoplasmic C-MYC & 64 & & & & $.000^{* *}$ \\
\hline- & $19(29.7 \%)$ & $11(17.2 \%)$ & $7(10.9 \%)$ & $1(1.6 \%)$ & \\
\hline+ & $25(39.1 \%)$ & $6(9.4 \%)$ & $17(26.6 \%)$ & $2(3.1 \%)$ & \\
\hline++ & $20(31.2 \%)$ & $3(4.7 \%)$ & $6(9.4 \%)$ & $11(17.2 \%)$ & \\
\hline Nuclear C-MYC (\%) & 64 & $16 \pm 20$ & $11 \pm 13$ & $16 \pm 19$ & .537 \\
\hline P53 (\%) & 61 & $19 \pm 21$ & $13 \pm 17$ & $30 \pm 31$ & .082 \\
\hline$C D 8+T I L\left(/ 0.2 \mathrm{~mm}^{2}\right)$ & 61 & $160.00 \pm 55.52$ & $132.07 \pm 54.60$ & $105.71 \pm 45.69$ & $.020^{*}$ \\
\hline$P D-L 1$ & 48 & & & & .599 \\
\hline- & $15(31.3 \%)$ & $6(12.5 \%)$ & $6(12.5 \%)$ & $3(6.3 \%)$ & \\
\hline+ & $28(58.3 \%)$ & $4(8.3 \%)$ & $17(35.4 \%)$ & $7(14.6 \%)$ & \\
\hline++ & $4(8.3 \%)$ & $1(2.1 \%)$ & $2(4.2 \%)$ & $1(2.1 \%)$ & \\
\hline+++ & $1(2.1 \%)$ & $0(0.0 \%)$ & $1(2.1 \%)$ & $0(0.0 \%)$ & \\
\hline SOX11 (\%) & 64 & $33 \pm 31$ & $29 \pm 31$ & $29 \pm 27$ & .887 \\
\hline
\end{tabular}


Table 2 (on next page)

Clinical and immunohistochemical staining characteristics of patients grouped by treatment response

Note: $* P<0.05$. 


\begin{tabular}{|c|c|c|c|c|c|}
\hline Variable & $\mathbf{n}$ & $\mathbf{C R}$ & PR & PD & $\begin{array}{c}P \\
\text { value } \\
\end{array}$ \\
\hline Sex & & & & & .117 \\
\hline Male & $23(74.2 \%)$ & $5(16.1 \%)$ & $10(32.3 \%)$ & $8(25.8 \%)$ & \\
\hline Female & $8(25.8 \%)$ & $0(0.0 \%)$ & $7(22.6 \%)$ & $1(3.2 \%)$ & \\
\hline Age (years) & 31 & $52.60 \pm 3.91$ & $56.24 \pm 12.73$ & $56.89 \pm 8.89$ & .760 \\
\hline$W B C\left(10^{9} / L\right)$ & 31 & $10.06 \pm 3.61$ & $7.68 \pm 3.78$ & $7.54 \pm 3.43$ & .407 \\
\hline$L D H(U / L)$ & 31 & $334.82 \pm 243.60$ & $333.21 \pm 269.65$ & $273.31 \pm 95.30$ & .804 \\
\hline ECOG performance & & & & & 0.130 \\
\hline \multicolumn{6}{|l|}{ Status } \\
\hline $\mathbf{0}$ & $21(67.7 \%)$ & $4(12.9 \%)$ & $13(41.9 \%)$ & $4(12.9 \%)$ & \\
\hline $\mathbf{1}$ & $8(25.8 \%)$ & $1(3.2 \%)$ & $2(6.5 \%)$ & $5(16.1 \%)$ & \\
\hline 2 & $2(6.5 \%)$ & $0(0.0 \%)$ & $2(6.5 \%)$ & $0(0.0 \%)$ & \\
\hline Ann Arbor stage & & & & & .905 \\
\hline II & $1(3.2 \%)$ & $0(0.0 \%)$ & $1(3.2 \%)$ & $0(0.0 \%)$ & \\
\hline III & $16(51.6 \%)$ & $3(9.7 \%)$ & $8(25.8 \%)$ & $5(16.1 \%)$ & \\
\hline IV & $14(45.2 \%)$ & $2(6.5 \%)$ & $8(25.8 \%)$ & $4(12.9 \%)$ & \\
\hline Location & & & & & .437 \\
\hline Nodal & $23(74.2 \%)$ & $3(9.7 \%)$ & $12(38.7 \%)$ & $8(25.8 \%)$ & \\
\hline Extra-nodal & $8(25.8 \%)$ & $2(6.5 \%)$ & $5(16.1 \%)$ & $1(3.2 \%)$ & \\
\hline B symptoms & & & & & .844 \\
\hline No & $15(48.4 \%)$ & $3(9.7 \%)$ & $8(25.8 \%)$ & $4(12.9 \%)$ & \\
\hline Yes & $16(51.6 \%)$ & $2(6.5 \%)$ & $9(29.0 \%)$ & $5(16.1 \%)$ & \\
\hline Risk group & & & & & .720 \\
\hline low & $11(35.5 \%)$ & $2(6.5 \%)$ & $6(19.4 \%)$ & $3(9.7 \%)$ & \\
\hline intermediate & $16(51.6 \%)$ & $3(9.7 \%)$ & $8(25.8 \%)$ & $5(16.1 \%)$ & \\
\hline high & $4(12.9 \%)$ & $0(0.0 \%)$ & $3(9.7 \%)$ & $1(3.2 \%)$ & \\
\hline Cytoplasmic C-MYC & & & & & $.031^{*}$ \\
\hline- & $11(35.5 \%)$ & $2(6.5 \%)$ & $8(25.8 \%)$ & $1(3.2 \%)$ & \\
\hline+ & $11(35.5 \%)$ & $3(9.7 \%)$ & $2(6.5 \%)$ & $6(19.4 \%)$ & \\
\hline++ & $9(29.0 \%)$ & $0(0.0 \%)$ & $7(22.6 \%)$ & $2(6.5 \%)$ & \\
\hline Nuclear C-MYC (\%) & 31 & $17 \pm 12$ & $15 \pm 17$ & $6 \pm 7$ & .227 \\
\hline P53 (\%) & 31 & $16 \pm 21$ & $20 \pm 23$ & $20 \pm 23$ & .950 \\
\hline$C D 8+T I L\left(/ 0.2 \mathrm{~mm}^{2}\right)$ & 31 & $154.00 \pm 35.78$ & $145.00 \pm 55.74$ & $111.11 \pm 64.51$ & .277 \\
\hline$P D-L 1$ & & & & & .352 \\
\hline- & $11(45.8 \%)$ & $1(4.2 \%)$ & $5(20.8 \%)$ & $5(20.8 \%)$ & \\
\hline+ & $11(45.8 \%)$ & $3(12.5 \%)$ & $6(25.0 \%)$ & $2(8.3 \%)$ & \\
\hline++ & $2(8.3 \%)$ & $0(0.0 \%)$ & $2(8.3 \%)$ & $0(0.0 \%)$ & \\
\hline SOX11 (\%) & 31 & $36 \pm 45$ & $26 \pm 30$ & $34 \pm 31$ & .757 \\
\hline Ki67 (\%) & 31 & $38 \pm 16$ & $50 \pm 27$ & $38 \pm 30$ & .480 \\
\hline
\end{tabular}




\section{Table 3(on next page)}

Correlation coefficient analysis for different parameters with risk group

Note: $* P<0.05, * * P<0.01$. 
1

\begin{tabular}{lccc}
\hline \multicolumn{1}{c}{ Variable } & n & $\begin{array}{c}\text { Spearman correlation coefficient } \\
\text { with risk group }\end{array}$ & P value \\
\hline Sex & 64 & 0.055 & 0.669 \\
Age & 64 & $0.454^{* *}$ & 0.000 \\
WBC & 64 & $0.273^{*}$ & 0.029 \\
LDH & 64 & $0.593^{* *}$ & 0.000 \\
ECOG performance status & 64 & 0.207 & 0.101 \\
Ann Arbor stage & 64 & 0.078 & 0.542 \\
Location & 64 & 0.085 & 0.502 \\
B symptoms & 64 & 0.138 & 0.278 \\
Response & 31 & 0.058 & 0.755 \\
Cytoplasmic C-MYC & 64 & $0.496^{* *}$ & 0.000 \\
Nuclear C- MYC & 64 & 0.005 & 0.968 \\
P53 & 61 & 0.079 & 0.544 \\
CD8+TIL & 61 & $-0.351^{* *}$ & 0.006 \\
PD-L1 & 48 & 0.163 & 0.268 \\
SOX11 & 64 & -0.020 & 0.873 \\
Ki67 & 64 & $0.303^{*}$ & 0.015 \\
\hline
\end{tabular}

2 